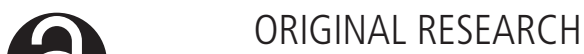

\section{Supervised pulmonary tele-rehabilitation versus pulmonary rehabilitation in severe COPD: a randomised multicentre trial}

\author{
Henrik Hansen, ${ }^{1}$ Theresa Bieler, ${ }^{2}$ Nina Beyer, ${ }^{3}$ Thomas Kallemose, $^{4}$ Jon Torgny Wilcke, ${ }^{5}$ \\ Lisbeth Marie Østergaard ${ }_{1}^{6}$ Helle Frost Andeassen, ${ }^{6}$ Gerd Martinez, ${ }^{7}$ Marie Lavesen, ${ }^{8}$ \\ Anne Frølich, ${ }^{9}$ Nina Skavlan Godtfredsen ${ }^{3,7}$
}

\begin{abstract}
- Additional material is published online only. To view please visit the journal online (http://dx.doi.org/10.1136/ thoraxjnl-2019-214246).

For numbered affiliations see
\end{abstract} end of article.

\section{Correspondence to} Dr Henrik Hansen, Respiratory Research Unit, Department of Respiratory Medicine, Hvidovre Hospital, Hvidovre, Denmark; henrik.hansen.09@regionh.dk

Received 24 October 2019 Revised 14 February 2020 Accepted 3 March 2020 Published Online First 30 March 2020

Check for updates

(C) Author(s) (or their employer(s)) 2020. Re-use permitted under CC BY-NC. No commercial re-use. See rights and permissions. Published by BMJ.

To cite: Hansen $\mathrm{H}$, Bieler $\mathrm{T}$ Beyer $\mathrm{N}$, et al. Thorax 2020:75:413-421.

\section{ABSTRACT}

Rationale Pulmonary rehabilitation (PR) is an effective, key standard treatment for people with COPD. Nevertheless, low participant uptake, insufficient attendance and high drop-out rates are reported. Investigation is warranted of the benefits achieved through alternative approaches, such as pulmonary telerehabilitation (PTR).

Objective To investigate whether PTR is superior to conventional PR on 6 min walk distance (6MWD) and secondarily on respiratory symptoms, quality of life, physical activity and lower limb muscle function in patients with COPD and $\mathrm{FEV}_{1}<50 \%$ eligible for routine hospital-based, outpatient PR.

Methods In this single-blinded, multicentre, superiority randomised controlled trial, patients were assigned 1:1 to 10 weeks of groups-based PTR (60 min, three times weekly) or conventional PR ( 90 min, two times weekly). Assessments were performed by blinded assessors at baseline, end of intervention and at 22 weeks' follow-up from baseline. The primary analysis was based on the intention-to-treat principle.

Measurements and main results The primary outcome was change in 6MWD from baseline to 10 weeks; 134 participants (74 females, mean \pm SD age $68 \pm 9$ years, $F E V, 33 \% \pm 9 \%$ predicted, $6 \mathrm{MWD} 327 \pm 103$ metres) were included and randomised. The analysis showed no between-group differences for changes in 6MWD after intervention (9.2 metres ( $95 \% \mathrm{Cl}:-6.6$ to $24.9))$ or at 22 weeks' follow-up (-5.3 metres $(95 \% \mathrm{Cl}$ : -28.9 to 18.3$)$ ). More participants completed the PTR intervention $(n=57)$ than conventional PR $(n=43)\left(\chi^{2}\right.$ test $\mathrm{p}<0.01$ )

Conclusion PTR was not superior to conventional PR on the 6MWD and we found no differences between groups. As more participants completed PTR, supervised PTR would be relevant to compare with conventional PR in a non-inferiority design.

Trialregistration number

ClinicalTrials.gov(NCT02667171), 28 January 2016.

\section{INTRODUCTION}

Pulmonary rehabilitation (PR) is recognised as an important, standard treatment for people with chronic obstructive pulmonary disease (COPD). $\mathrm{PR}$ is well documented to reduce symptoms and increase walking capacity and quality of life (QoL), but its effect on physical activity level (PAL) is

\section{Key messages}

What is the key question?

- Can a supervised pulmonary tele-rehabilitation programme, including structured exercise and education, deliver higher programmeadherence and thereby superior benefits to a conventional hospital-based pulmonary rehabilitation (PR) programme for patients with severe COPD?

What is the bottom line?

- This pulmonary tele-rehabilitation model demonstrated short-term and medium-term improvements in functional capacity and disease-related symptoms that were not superior to conventional hospital-based PR for patients with severely progressed COPD.

Why read on?

- Despite the benefits, PR programmes are challenged by low participant uptake, insufficient attendance and high drop-out rates; supervised pulmonary tele-rehabilitation may be a useful second-line option to improve access for patients with severe COPD who cannot participate in or comply with a conventional hospital-based PR programme.

limited..$^{1-5}$ Despite the benefits, PR programmes are challenged by low participant uptake, insufficient attendance and high drop-out rates. ${ }^{6-9}$ Barriers have previously been reported, including transportation issues, symptom severity, acute exacerbations, lack of energy and disruption of daily routines. ${ }^{6} 1011$ Recently, the American Thoracic Society (ATS)/European Respiratory Society (ERS) recommended investigating alternative approaches to PR, such as tele-rehabilitation, in an attempt to increase uptake and make PR available to more patients. ${ }^{12}$ To date, two randomised controlled trials (RCTs) have explored the effect of unsupervised web-based or video-demonstrated individual exercise and education compared with conventional group-based PR in patients with stable moderateto-severe COPD. ${ }^{13} 14$ The studies did not find differences between interventions for outcomes of walking capacity and respiratory symptoms. ${ }^{13} 14$ 
One study including patients with COPD and comorbid heart failure (NYHA II-IV) compared home-monitored exercise and weekly individual telephone supervision with usual care (no intervention). The intervention provided clinically relevant differences in walking capacity, respiratory symptoms and QoL compared with usual care. Furthermore per-protocol analyses showed that the gains were maintained at the 2-month follow-up. ${ }^{15}$ Lastly, in patients with moderate COPD, one small RCT study ( $\mathrm{n}=37$ ) compared usual care (no intervention) with a supervised pulmonary tele-rehabilitation (PTR) programme in groups of two to four patients. ${ }^{16}$ This study showed significant and clinically relevant between-group difference on endurance shuttle walk, anxiety and depression symptoms and self-efficacy in favour of supervised PTR. ${ }^{16}$ All four studies are limited in their conclusions as none declared a priori-design (superior, equivalence, non-inferior) in the trial registry protocol, and just two studies stated a hypothesis. ${ }^{14} 16$ Although results from the recent PTR studies are promising, effects from supervised PTR in groups compared with conventional outpatient supervised $\mathrm{PR}$ in groups remain to be investigated. Consequently, evidence is needed to ascertain any possible effect of a fully supervised real-time PTR programme on relevant outcomes. To our knowledge, our study is the first RCT investigating the short-term and medium-term effect of a supervised PTR programme compared with a supervised conventional PR programme on walking capacity, symptoms, QoL and PAL in patients with COPD, FEV $<50 \%$ and a high symptom burden eligible for routine hospitalbased outpatient PR. We hypothesised that the supervised PTR programme would be superior to a supervised conventional PR regarding change in $6 \mathrm{~min}$ walk distance (6MWD) because of an expected higher adherence rate, leading to a greater response. ${ }^{17}$ This paper reports on the clinical outcomes. A full economic analysis will be published separately.

\section{METHODS}

\section{Trial design and participants}

We conducted a randomised clinical, assessor-blinded and statistician-blinded, superiority, multicentre trial with two parallel groups to investigate the effect of supervised PTR compared with conventional PR on walking capacity in patients eligible for outpatient hospital-based PR. Patients were recruited from the respiratory departments of eight different university hospitals in Greater Copenhagen during March 2016 to October 2017. Inclusion and exclusion criteria corresponded to the criteria for outpatient hospital-based routine PR in the Capital Region of Copenhagen, Denmark, and pertained to adults with a clinical diagnosis of COPD defined as FEV1/FVC $<0.70$, FEV1 $<50 \%$, Medical Research Council $\geq 2$ and no participation in PR within 6 months of the start of intervention. ${ }^{17}{ }^{18}$ All patients provided written and verbal informed consent.

\section{Randomisation and blinding}

After baseline assessments, patients were randomly allocated 1:1 to receive PTR or conventional hospital-based PR. The allocation followed a computer-generated randomisation list made by a biostatistician for each recruiting hospital; treatment was denoted as A and B to ensure blinding of the biostatistician. A senior manager from an independent research department was responsible for the randomisation list and provided the draw to ensure concealment. All assessors were blinded to group allocation, hypotheses and intervention details. Patients were not possible to blind for allocation. In the case of failure to keep the assessor blinded, a second assessor was available to conduct the blinded assessment on another day. The biostatistician had the main responsibility for the data analyses.

\section{Intervention}

\section{Pulmonary tele-rehabilitation}

The details and appropriate dosage set-up from both intervention programs are available in the online supplementary material and in our previously published protocol article. ${ }^{17}$ In brief, the PTR programme was designed by the study group and aligned with exercise intensities and education themes from conventional PR. It was a group-based, supervised and standardised programme performed by the patients in their homes three times weekly for 10 weeks via a videoconference software system installed on a single touch screen. The exercise sessions lasted $35 \mathrm{~min}$ (weekly exercise volume $105 \mathrm{~min}$ ) with incorporated warm-up and high repetitive time-based muscle endurance training followed by $5 \mathrm{~min}$ ' rest before beginning a patient education session of $20 \mathrm{~min}$ (weekly education volume $60 \mathrm{~min}$ ).

\section{Conventional pulmonary rehabilitation}

The conventional outpatient hospital-based PR programme was group-based, supervised and standardised and was performed twice a week for 10 weeks (in one hospital, for 12 weeks). The programme followed the Danish Health Authority's National Clinical Guideline and the Regional Guidelines. ${ }^{171920}$ The exercise sessions lasted $60 \mathrm{~min}$ and incorporated warm-up, endurance and resistance training and a cool-down period (weekly exercise volume $120 \mathrm{~min}$ ). The patient education sessions lasted 60 to $90 \mathrm{~min}$ and took place once a week after the exercise session (detailed online supplementary available).

\section{Outcomes}

Full details on outcome, assessment procedures and quality control are available in the online supplementary material. Briefly, the primary outcome was change in the 6MWD on completion of the programme. Secondary outcomes were COPD Assessment Test (CAT), Hospital Anxiety and Depression Scale (HADS), EuroQol 5-Dimension Questionnaire (EQ-5D), the 30 s sit-to-stand test (30sec-STS), Clinical COPD Questionnaire (CCQ) and Physical Activity Level (PAL). The PAL was measured with activePAL triaxial accelerometer (PAL Technologies Ltd, Glasgow, UK) worn 24 hours for 5 days. PAL was measured on 73 patients residing within a radius of $25 \mathrm{~km}$ of Bispebjerg University Hospital. All assessment procedures were performed at baseline, end of intervention and at 22 weeks' follow-up from baseline. The procedures were reproducible and have been published. $^{21}$ Descriptive variables included body mass index, smoking status, medication and Charlson Comorbidity Index, spirometry and anthropometric measures, which followed the standardised protocols from the Danish Society of Respiratory Medicine. ${ }^{22}$ Adverse events, hospitalisations and deaths were recorded throughout the trial by the National Health Data Authorities.

\section{Statistical analysis}

For the $6 \mathrm{MWD}$, a change of $26 \mathrm{~m}$ is considered a minimal clinically important difference (MCID) in patients with COPD and FEV1 $<50 \% .^{23-25}$ Based on a two-sample independent t-test with an MCID of $26 \mathrm{~m}$, a SD of $44.6 \mathrm{~m},{ }^{24}$ a power of $80 \%$, a significance level of 0.05 and an anticipated drop-out rate of 30\%, 134 patients were recruited. Using this sample size, expected SD and existing MCID, power estimations for the secondary outcomes revealed $80 \%$ power to detect MCID in all 
secondary outcomes except CCQ and PAL. ${ }^{17}$ Descriptive data for the PTR and conventional PR are presented as mean and SD for continuous variables and frequency for categorical variables. Differences between the intervention groups in change of primary and secondary outcomes (end of intervention - baseline and 22 weeks' follow-up - baseline) were analysed by mixed effect models. The models included adjustment for treatment group, age, sex, body mass index, $\mathrm{FEV}_{1}$, Charlson Comorbidity Index, smoking status and a random effect for hospital allocation. To account for possible regression to the mean effect, the baseline measure for the outcome was also included as a fixed effect variable in the models. Normal distribution of the model residuals was evaluated by Q-Q plots. All data are considered missing at random, using this with the likelihood-estimation in the mixed effect model, the ignorability assumption for the likelihood estimator is used to account for missing data in the model estimates ${ }^{26}$ (number of data sets is stated in tables 1 and 2 and online supplementary tables S2 and S3). Group differences on number of patients remaining in their programmes for the full intervention period, adherence, hospitalisation and death were analysed with $\chi^{2}$ test. Adherence/attendance was defined as a patient participating in an entire scheduled exercise and education session. Analysis of age and sex differences between patients with and without outcome measures was done by $\chi^{2}$ and Wilcoxon rank-sum test. Per-protocol analysis included patients attending $\geq 70 \%$ of the planned sessions. Statistical analyses were carried out using R 3.2.2 (R Foundation for Statistical Computing, Vienna, Austria). P values of less than 0.05 were considered statistically significant.

\section{RESULTS}

\section{Recruitment}

Of the patients suitable for hospital-based PR, 1099 met the inclusion criteria and were considered; 714 patients refused PR and were thus deemed ineligible. Of 385 eligible patients, the majority $(\mathrm{n}=251)$ wished to undertake conventional PR and declined participation in the study. One hundred and thirtyfour patients provided informed consent and were randomised ( $n=67$ in each group) (figure 1). Baseline characteristics are shown in table 1 .

\section{Primary outcome}

Tables 2 and 3 show the differences between and the changes within the groups at the end of PR/PTR and at the 22 weeks' follow-up from baseline. We found no statistically significant between-group difference for change in the 6MWD after intervention (table 2). Both groups demonstrated statistically significant improvements in the 6MWD after intervention, but the gain was sustained and significant only in the PTR group at 22 weeks' follow-up from baseline (table 3). None of the group improvements exceeded the MCID at any measurement time point.

\section{Secondary outcomes}

The between-group difference for changes in respiratory symptoms (CAT) was statistically different at the end of intervention with a greater symptom reduction difference of -1.6 points $(p=0.04)$ in the PTR group that did not exceed the MCID (table 2). There was no between-group difference at the 22 weeks' follow-up from baseline. The groups did not exceed the MCID in respiratory symptom reduction at any measurement time point (table 3). The PTR group had a statistically significant reduction in anxiety and depression scores (HADS-A and HADS-D) compared with the conventional PR group after intervention,

\begin{tabular}{|c|c|c|c|}
\hline Variables & All $(n=134)$ & PTR $(n=67)$ & PR $(n=67)$ \\
\hline Female sex, n (\%) & $74(55)$ & $35(52)$ & $39(58)$ \\
\hline Age, year & $68.3(9.0)$ & $68.4(8.7)$ & $68.2(9.4)$ \\
\hline Body mass index, kg/m² & $25.7(5.8)$ & $25.5(5.0)$ & $25.9(6.4)$ \\
\hline FEV1, \% predicted & $33.1(9.4)$ & $32.6(10.3)$ & $33.7(8.4)$ \\
\hline FEV1/FVC, \% & $43.3(11.2)$ & $43.9(11.3)$ & 42.7 (11.1) \\
\hline GOLD I/II/IIIIIV, \% & 0/0/61/39 & 0/0/55/45 & 0/0/67/33 \\
\hline $\mathrm{A} / \mathrm{B} / \mathrm{C} / \mathrm{D}, \%$ & $2 / 34 / 4 / 60$ & $5 / 34 / 3 / 58$ & $0 / 33 / 4 / 63$ \\
\hline LTOT, n (\%) & $20(15)$ & $11(16)$ & $9(13)$ \\
\hline Sp02 at rest, $\%$ & $94.6(2.8)$ & $94.6(2.4)$ & $94.8(3.1)$ \\
\hline MRC 1/2/3/4/5, n & 0/2/65/50/17 & $0 / 2 / 30 / 27 / 8$ & $0 / 0 / 35 / 23 / 9$ \\
\hline \multicolumn{4}{|l|}{ Smoking status, n (\%) } \\
\hline Never & $3(2)$ & $2(3)$ & $1(1)$ \\
\hline Former & $99(75)$ & $51(79)$ & $48(72)$ \\
\hline Current & $30(23)$ & $12(18)$ & $18(27)$ \\
\hline Pack-year history, mean (SD) & $43.5(20.2)$ & $42.4(23.1)$ & $44.5(17.3)$ \\
\hline BODE index points, median (IQR) & $5.0(4-6)$ & $5.0(4-7)$ & $5.0(4-6)$ \\
\hline 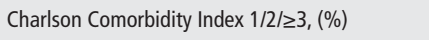 & $40 / 37 / 23$ & $45 / 40 / 15$ & $34 / 33 / 33$ \\
\hline Exacerbations, previous 12 month, (median, IQR) & $2(0-3)$ & $2(0-4)$ & $2(1-3)$ \\
\hline \multicolumn{4}{|l|}{ Current medication, n (\%) } \\
\hline SABA & $112(84)$ & $56(84)$ & $56(84)$ \\
\hline SABA + SAMA & $11(8)$ & $6(9)$ & $5(7)$ \\
\hline LABA & $2(1)$ & $1(1)$ & $1(1)$ \\
\hline LAMA & $3(2)$ & $2(3)$ & $1(1)$ \\
\hline LABA + LAMA & $24(18)$ & $12(18)$ & $12(18)$ \\
\hline$L A B A+I C S$ & $8(6)$ & $5(7)$ & $3(5)$ \\
\hline$L A B A+L A M A+I C S$ & $93(69)$ & $45(67)$ & $48(71)$ \\
\hline Oral steroids & $2(1)$ & $2(3)$ & $0(0)$ \\
\hline Walking aid, walker/other, n (\%) & $27 / 18$ (34) & $14 / 9(34)$ & 13/9 (33) \\
\hline Highest 6MWD, metre & $327.3(102.8)$ & $322.3(108.3)$ & $332.3(97.5)$ \\
\hline Highest 30sec-STS, repetitions & $9.8(4.3)$ & $9.9(4.7)$ & $9.6(3.8)$ \\
\hline \multicolumn{4}{|l|}{ Physical activity level ${ }^{*}$} \\
\hline Daily step count, steps & $3091(2161)$ & 2779 (1966) & $3422(2335)$ \\
\hline Time sedentary, min & $1205(133)$ & $1244(121)$ & 1164 (134) \\
\hline Time active, min & $235(133)$ & $196(121)$ & $276(134)$ \\
\hline CAT, score & $20.1(7.0)$ & $19.8(7.3)$ & $20.4(6.6)$ \\
\hline \multicolumn{4}{|l|}{ HADS, score } \\
\hline HADS-anxiety & $6.3(3.5)$ & $6.8(3.8)$ & $5.9(3.1)$ \\
\hline HADS-depression & $4.3(3.0)$ & $4.5(2)$. & $4.1(3.1)$ \\
\hline EQ-5D, VAS score & $52.7(19.2)$ & $51.5(19.4)$ & $53.9(19.1)$ \\
\hline EQ-5D, index score & $0.68(0.16)$ & $0.66(0.20)$ & $0.70(0.12)$ \\
\hline \multicolumn{4}{|l|}{ CCQ, score } \\
\hline Symptoms & $2.9(1.2)$ & $2.8(1.2)$ & $3.0(1.2)$ \\
\hline Functional & $2.9(1.2)$ & $2.8(1.1)$ & $3.0(1.3)$ \\
\hline Mental & $2.8(1.4)$ & $2.8(1.5)$ & $2.9(1.4)$ \\
\hline Total & $2.8(0.9)$ & $2.7(0.9)$ & $2.9(1.0)$ \\
\hline
\end{tabular}

Data are presented as mean (SD) except where otherwise indicated.

${ }^{*}$ ActivePAL triaxial accelerometer worn by in total 73 patients (PTR/PR: $\left.37 / 36\right)$. Any statistically significant difference between PTR and PR denoted ${ }^{*} \mathrm{p}<0.05$.

$A / B / C / D$, risk stratification, airflow obstruction, dyspnea and exercise capacity; BODE index, body mass index; CAT, COPD Assessment Test; CCQ, Clinical COPD Questionnaire; EQ-5D, Euro-Qol 5-dimension; FEV1, forced expiratory volume in the first second; FVC, forced vital capacity; GOLD, Global initiative for Chronic Obstructive Lung Disease; HADS, Hospital Anxiety and Depression Score; ICS, inhaled corticosteroid; LABA, long-acting $\beta 2$-agonist; LAMA, long-acting muscarinic antagonist; LTOT, longterm oxygen therapy; MRC, Medical Research Council; 6MWD, 6 min walk distance; PR, pulmonary rehabilitation; PTR, pulmonary tele-rehabilitation; SABA, short-action $\beta 2$-agonist; SAMA, short-acting muscarinic antagonist; 30-sec STS, 30 s sit-to-stand test; $\mathrm{SpO2}$, arterial oxygen saturation as measured by pulse oximetry; VAS, Visual Analogue Scale. 
Table 2 Between-group differences in primary and secondary outcomes in PTR and PR groups. Intention-to-treat principle

\begin{tabular}{|c|c|c|c|c|}
\hline & \multicolumn{4}{|c|}{ Between-group differences from baseline $(95 \% \mathrm{Cl})$} \\
\hline & \multicolumn{2}{|l|}{ PR-PTR (unadjusted) } & \multicolumn{2}{|l|}{ PR-PTR (adjusted) } \\
\hline & End rehabilitationt & 22 weeks from baseline $\neq$ & End rehabilitationt & 22 weeks from baseline $\ddagger$ \\
\hline 6MWD, min & $6.3(-9.8$ to 22.5$)$ & $-11.0(-34.4$ to 12.4$)$ & $8.3(-7.7$ to 24.3$)$ & $-3.9(-27.9$ to 19.9$)$ \\
\hline \multicolumn{5}{|l|}{ Secondary outcomes } \\
\hline \multicolumn{5}{|l|}{ HADS } \\
\hline Anxiety, points & $1.1(0.1 \text { to } 2.1)^{*}$ & $0.2(-0.9$ to 1.4$)$ & $1.2(0.2 \text { to } 2.3)^{*}$ & $0.4(-0.8$ to 1.6$)$ \\
\hline Depression, points & $0.7(-0.1$ to 1.5$)$ & $-0.2(-1.3$ to 1.0$)$ & $0.9(0.1 \text { to } 1.7)^{*}$ & $-0.2(-1.3$ to 1.0$)$ \\
\hline EQ-5D-VAS, points & $-0.2(-6.4$ to 5.9$)$ & $0.8(-5.8$ to 7.5$)$ & $-0.2(-6.2$ to 5.9$)$ & $1.6(-5.1$ to 8.3$)$ \\
\hline Symptoms, points & $0.2(-0.1$ to 0.5$)$ & $0.1(-0.3$ to 0.5$)$ & $0.2(-0.1$ to 0.5$)$ & $0.1(-0.2$ to 0.5$)$ \\
\hline Total, points & $0.2(-0.1$ to 0.4$)$ & $0.1(-0.2$ to 0.3$)$ & $0.2(-0.1$ to 0.5$)$ & $0.1(-0.1$ to 0.4$)$ \\
\hline \multicolumn{5}{|l|}{ PAL } \\
\hline Steps per day & $-283(-845$ to 278$)$ & $-302(-1035$ to 419$)$ & $-436(-1010$ to 138$)$ & $-103(-886$ to 597$)$ \\
\hline Sedentary, $\min$ & $9.4(-35.2$ to 51.3$)$ & $8.6(-53.8$ to 36.6$)$ & 7.7 (-49.0 to 52.2$)$ & $14.6(-32.5$ to 58.5$)$ \\
\hline Active, min & $-9.4(-51.3$ to 35.4$)$ & $-8.6(-36.6$ to 53.8$)$ & $-7.7(-52.2$ to 49.0$)$ & $-14.6(-58.5$ to 32.5$)$ \\
\hline
\end{tabular}

Data are mean difference $(95 \% \mathrm{Cl})$.

${ }^{*} \mathrm{P}$ value for group mean change differences $<0.05$.

tComplete observations ( $n$ ) used for the likelihood estimate from end of rehabilitation to baseline (total): 6MWD: (115); 30sec-STS: (115); CAT: (119); HADS: (110); EQ-5d-VAS: (119); CCQ: (119); PAL: (59).

$\ddagger$ Complete observations ( $n$ ) used for the likelihood estimate from 22 weeks' follow-up from baseline to baseline (total): 6MWD: (95); 30sec-STS: (95); CAT: (106); HADS: (100); EQ-5d-VAS: (104); CCQ: (106); PAL: (55).

CAT, COPD Assessment Test; CCQ, COPD Clinical Questionnaire; EQ-5D, EuroQol 5-Dimension Questionnaire; HADS, Hospital Anxiety and Depression Scale; $6 \mathrm{MWD}, 6$ min walk distance; PAL, physical activity level; PR, pulmonary rehabilitation; PTR, pulmonary tele-rehabilitation; 30sec-STS, 30 s sit-to-stand test; VAS, Visual Analogue Scale.

but it did not exceed the MCID. There were no between-group differences at the 22-week follow-up (table 2). The within group improvements on the anxiety domain were significant for the PTR group after intervention but did not exceed the MCID and the improvement was not sustained at the 22-week follow-up. No group exceeded the MCID for QoL (EQ-5D-VAS) and lower limb muscle function (30sec-STS) (table 3). We registered a statistically significant decrease in number of daily steps per day in the PR group from baseline to end of intervention and to the 22-week follow-up, whereas daily steps per day remained unchanged in the PTR group (table 3). There was no difference between groups in the per-protocol analyses in any outcome at any measurement time point (see online supplementary tables S2 and S3).

The attendance rate was a median of 25 session (IQR: 20 to 28 ) in the PTR group and 16 session (IQR: 8 to 19 ) in the PR group and thus the exercise volume was a median of $750 \mathrm{~min}$ (IQR: 600 to 840) in the PTR group and $960 \mathrm{~min}$ (IQR: 480 to 1140 ) in the PR group. A significantly higher number of patients remained in the PTR programme for the full intervention period compared with the PR programme (PTR: 57/67 vs PR: 43/67; OR: 3.18 (95\% CI: 1.37 to 7.35$), \mathrm{p}<0.01)$. However, there was no difference between groups for those who attended $\geq 70 \%$ of the programs' total sessions, (PTR: 49/67 vs PR: 42/67; OR: 1.68 (95\% CI: 0.78 to 3.37), $\mathrm{p}<0.27$ ). The mean adherence rate among drop-outs who attended at least one session was $50 \%$ of all sessions (IQR\%: 42 to 64 ) in the PTR programme versus $33 \%$ of all sessions (IQR\%: 18 to 49 ) in the PR programme.
Two drop-outs, both in the PR group, were potentially related to adverse effects of the PR programme. Both events were related to overload with subsequent pain in the knee and groin, respectively, and did not require medical treatment. In total, 41 hospital admissions related to COPD exacerbations were recorded (PTR: $n=21 ; P R: n=20 ; p=0.77$ ) during the rehabilitation period, and 74 hospitalisations related to COPD exacerbations (PTR: $n=38 ; P R: n=36 ; p=0.97$ ) were recorded at the 22-week follow-up (see the online supplementary $\mathrm{S}$ for diagnostic codes used in the registry from the National Health Data Authorities and online supplementary S5 for hospital days and outpatient visits). There was no significant difference between groups for all cause hospitalisations during rehabilitation. Three deaths (PTR: $\mathrm{n}=1$; PR: $\mathrm{n}=2$ ) occurred during the rehabilitation period, and another three had died at the 22-week follow-up $(\mathrm{p}=1.0)$.

No difference could be shown between patients with and without missing outcome measurement on sex, all $\mathrm{p}$ values $>0.07$. By contrast, the median age was significantly higher among patients with missing values for 6MWD, 30sec-STS, repetitions and CCQ mental score.

\section{Registered problems with the technical solution}

Major technical issues leading to cancellation and rescheduling of group sessions affected 2 of 360 group sessions. Minor temporary technical issues (ie, sound artefacts, screen freezes) not leading to cancellation or delay were present in $14 \%$ of the total 


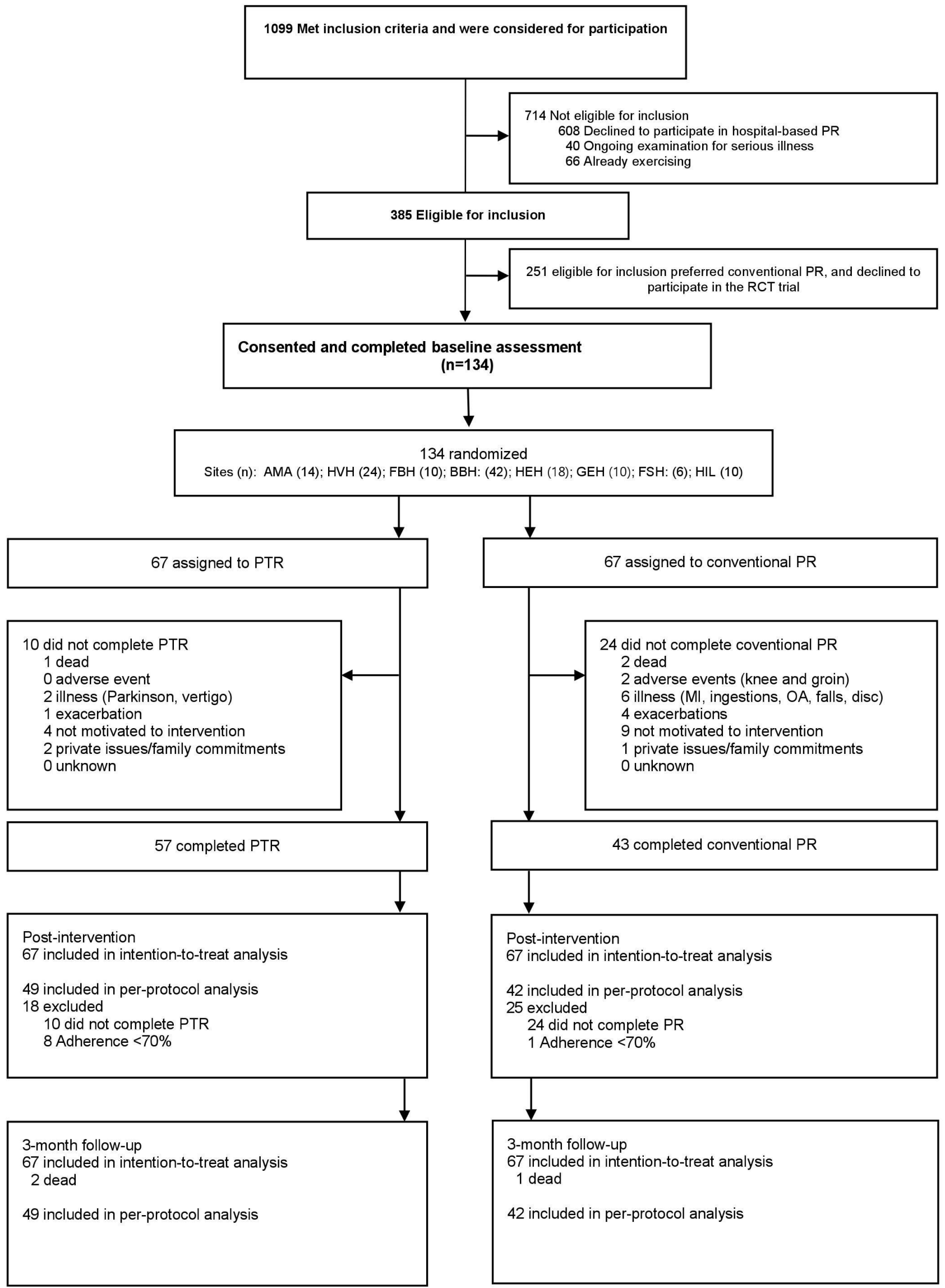

Figure 1 Consolidated Standards of Reporting Trials diagram. AMA, Amager; BBH, Bispebjerg; Disc, discogenic issue; FBH, Frederiksberg; FSH, Frederikssund; GEH, Gentofte; HEH, Herlev; HIL, Hillerød; HVH, Hvidovre; MI, myocardial infarction; OA, osteoarthritis; PR, pulmonary rehabilitation; PTR, pulmonary tele-rehabilitation, RCT, randomisedcontrolled trial. 
Table 3 Within-group changes in primary and secondary outcomes in PTR and PR groups. Intention-to-treat principle

\begin{tabular}{|c|c|c|c|c|}
\hline & \multicolumn{4}{|c|}{ Within-group changes from baseline $(95 \% \mathrm{Cl})$} \\
\hline & \multicolumn{2}{|l|}{$\operatorname{PTR}(n=67)$} & \multicolumn{2}{|l|}{$\operatorname{PR}(n=67)$} \\
\hline & End rehabilitationt & 22 weeks from baseline & End rehabilitationt & 22 weeks from baseline $\ddagger$ \\
\hline $6 \mathrm{MWD}, \min$ & $17.2(5.8 \text { to } 28.5)^{*}$ & $22.0(5.0 \text { to } 39.1)^{*}$ & $23.5(12.1 \text { to } 35.0)^{*}$ & $11.0(-5.2$ to 27.2$)$ \\
\hline \multicolumn{5}{|l|}{ Secondary outcomes } \\
\hline \multicolumn{5}{|l|}{ HADS } \\
\hline Anxiety, points & $-1.0(-1.7 \text { to }-0.2)^{*}$ & $-0.5(-1.4$ to 0.5$)$ & $0.1(-0.6$ to 0.8$)$ & $-0.3(-1.2$ to 0.7$)$ \\
\hline Depression, points & $-0.4(-1.1$ to 0.3$)$ & $0.5(-0.4$ to 1.5$)$ & $0.3(-0.4$ to 1.0$)$ & $0.3(-0.6$ to 1.4$)$ \\
\hline EQ5D-VAS, points & $3.2(-1.2$ to 7.6$)$ & $3.5(-1.2$ to 8.2$)$ & $2.9(-1.4$ to 7.2$)$ & $4.2(-0.4$ to 9.0$)$ \\
\hline Symptoms, points & $-0.3(-0.6 \text { to }-0.1)^{*}$ & $-0.2(-0.5$ to 0.1$)$ & $-0.2(-0.4$ to 0.1$)$ & $0.1(-0.3$ to 0.5$)$ \\
\hline Total, points & $-0.3(-0.4 \text { to }-0.1)^{*}$ & $0.0(-0.2$ to 0.2$)$ & $-0.1(-0.3$ to 0.1$)$ & $0.1(-0.2$ to 0.3$)$ \\
\hline \multicolumn{5}{|l|}{ PAL } \\
\hline Steps per day & $-116(-503$ to 270$)$ & $-292(-852$ to 307$)$ & $-400(-803 \text { to }-2.3)^{*}$ & $-594(-1164 \text { to }-57)^{*}$ \\
\hline Sedentary, min & $29.0(-29.9$ to 95.4$)$ & $18.8(-11.8$ to 49.3$)$ & 38.3 (-21.7 to 107.3$)$ & $10.1(-21.0$ to 41.3$)$ \\
\hline Active, min & -29.0 (-95.4 to 29.9$)$ & $-18.8(-49.3$ to 11.8$)$ & $-38.3(-107.3$ to 21.7$)$ & $-10.1(-41.3$ to 21.0$)$ \\
\hline
\end{tabular}

Data are mean difference $(95 \% \mathrm{Cl})$. Estimates adjusted for baseline outcome measure. Estimates calculated for baseline measure equal to the mean baseline measure for study population.

${ }^{*} \mathrm{P}$ value within group changes $<0.05$.

tComplete observations ( $\mathrm{n}$ ) used for the likelihood estimate from end of rehabilitation to baseline (PTR/PR): 6MWD: (56/59); 30-sec STS: (56/59); CAT: (59/62); HADS: (53/57); EQ5d-VAS: (57/62); CCQ: (57/62); PAL: (30/29).

‡Complete observations ( $\mathrm{n})$ used for the likelihood estimate from 22 weeks' follow-up from baseline to baseline (PTR/PR): 6MWD: (44/51); 30sec-STS: (44/51); CAT: (53/53); HADS: (50/50); EQ5d-VAS: (51/53); CCQ: (53/53); PAL: (28/27).

CAT, COPD Assessment Test; CCQ, COPD Clinical Questionnaire; EQ-5D, EuroQol 5-Dimension Questionnaire; HADS, Hospital Anxiety and Depression Scale; 6MWD, 6 min walk distance; PAL, physical activity level; PR, pulmonary rehabilitation; PTR, pulmonary tele-rehabilitation; 30sec-STS, 30 s sit-to-stand test; VAS, Visual Analogue Scale.

group session (49/360). Individual patient cancellation caused by technical problems was 12 of 1902 individual connections.

\section{DISCUSSION}

The main finding of this multicentre, single-blinded, randomised clinical trial was that supervised PTR was not superior to conventional hospital-based PR regarding walking capacity (6MWD). More patients completed PTR than $\mathrm{PR}$, whereas, contrary to our pre-hypothesis, there was no between-group difference in adherence rate (attending $\geq 70 \%$ of the planned sessions).

To our knowledge, the effects of a supervised PTR programme compared with a supervised conventional PR programme have not been previously investigated. Tsai et al found a clinically relevant effect on 6MWD and endurance shuttle walk test (ESWT) from supervised PTR compared with no intervention. ${ }^{16}$ The study by Bernocchi et al ${ }^{15}$ including patients with both COPD and heart failure reported superiority on 6MWD from an individual home-monitored exercise programme with a weekly phone call compared with no intervention; the intervention group exceeded the MCID and maintained the gain at the 2-month follow-up. ${ }^{15}$ The studies by Bourne et $a l^{13}$ and Chaplin $e t a l^{14}$ compared the effect of unsupervised web-based or video-demonstrated individual exercise and education with conventional group PR and found comparable between-group effects on walking tests and within-group changes that exceeded the MCID for 6MWD and ESWT but not for incremental shuttle walk test. ${ }^{13} 14$

By contrast, we found that neither conventional PR nor PTR improved the 6MWD above the MCID. Differences in population characteristics could in part explain our negative result. Compared with the above-mentioned studies, patients in our cohort had lower $\mathrm{FEV}_{1}$, higher symptom burden, more exacerbations, lower walking capacity and most likely more locomotor disadvantages because in our study, 34\% used a walking aid. Use of a walking aid or other indications of frailty have not been reported in the previous PTR studies. ${ }^{13-16}$ We recruited patients with identical real-world inclusion criteria for hospital-based PR, which could limit the consistency and efficacy of the results; however, our study reflects routine practice. Recently, two large RCTs by Holland et $a l^{27}$ and Horton et $a l^{28}$ including in total 453 patients with COPD, compared home-based PR with supervised centre-based PR, using a pragmatic trial design, also failed to achieve the expected MCID on walking capacity from both interventions. Finally, a retrospective cohort study of 2068 patients with COPD of differing severity and with different characteristics receiving gold standard outpatient or inpatient $\mathrm{PR}$ in the Netherlands reported that only $40 \%$ to $50 \%$ of all patients exceeded the MCID for 6MWD, HADS-A, HADS-D and St George's Respiratory Questionnaire, while the group average improvement almost exceeded the MCID. ${ }^{29}$ In this study, Spruit and colleagues demonstrated that patients respond 
very heterogeneously on both the physical and self-reported clinical outcomes. This suggests the need for reconsideration of the assumption that all patients with COPD are likely to respond similarly and sufficiently to specific and restricted primary outcomes in either conventional PR programs ${ }^{29}$ or alternative home-based programs. ${ }^{27} 28$

From the Cochrane review it appears that $43 \%$ of the larger studies (including more than 30 participants in each group) did not exceed the MCID for the $6 \mathrm{MWD}^{1}$ and, importantly, meta-epidemiological studies have shown that single-centre trials yield $14 \%$ to $27 \%$ larger effect sizes than do multicentre trials even when analyses are adjusted for sample size and bias. ${ }^{30}$

The completion rate in our study was significantly higher in the PTR group than in the PR group; however, we did not find a significant higher adherence rate in the PTR group (73\%) compared with the PR group (62\%). The drop-out rate of $36 \%$ in the PR group was anticipated and comparable to other studies reporting drop-out rates from $10 \%$ to $50 \% .^{671127} 2831$ The annual 2018 data audit from the Danish Regional Quality Database in COPD revealed that $45 \%$ of all participants in Danish outpatient hospital PR adhere to less than $50 \%$ of the PR program..$^{32}$ The real-world data from this quality database reflect the challenges with adherence in a conventional hospital-based real-world PR programme and should be contrasted with the distinctly higher adherence in the PTR programme, where 73\% of patients attended $\geq 70 \%$ of the sessions. Thus, PTR seemingly has the potential to overcome some barriers to adherence and completion. It should be noted that only one-third of patients eligible for this study were willing to participate in this RCT as they stated 'preferring conventional PR', thus limiting the external validity (figure 1). Patient preferences and motivation have a potential impact on the outcomes achieved in different settings. This indicates that PTR could be an alternative for some patients eligible for outpatient hospital-based PR. As the 134 patients who agreed to participate may be particularly motivated, the 608 patients who declined participation in conventional PR would be an important group of interest for future research in the field of exploring the relevance and effects of PTR as an alternative delivery model.

There were also a number of important secondary findings in the present study. We investigated a cohort of patients with an extremely low level of physical activity, with average steps per day corresponding to basal and limited activity, for example, getting out of bed, making a meal and infrequent walks outside the home. This low and unchanged PAL throughout the study could affect the outcome and explain why the MCID in 6MWD was not exceeded since PAL and steps above 7500 per day are considered essential for physical functioning and overall health. ${ }^{33}$

We found that the PTR group had a significant reduction in CAT, anxiety and depression scores (HADS-A and HADS-D) compared with the PR group after intervention; however, the reduction did not exceed the MCID and the difference was not persistent at the 22-week follow-up. The higher completion rate in the PTR group, where patients continued to receive realtime attention and care, could be a plausible explanation of the differences after intervention. The previously mentioned PTR studies by Chaplin et $a l^{14}$ and Bourne $e t a l^{13}$ did not find any between-group differences in HADS after intervention, while Tsai $e t a l^{16}$ found differences identical to ours with supervised PTR compared with no intervention. The impact of real-time supervision versus the web-based PTR, including the means of communication, is not possible to quantify but could potentially explain some of these inconsistent findings between the PTR studies.

We are not aware of any studies comparing non-supervised PTR with supervised PTR. Ability to navigate and interact independently on a tablet and a webpage was required in the non-supervised PTR studies, whereas for the patients in our study, it was sufficient to have naive technical ability and skills. ${ }^{1314}{ }^{17}$ Essentially, future tele-rehabilitation designs must include specific considerations regarding delivery form and content and technical skills of the targeted population, particularly if PTR is to be considered as an extended offer specifically to those who live remotely and to those who lack energy and resources to join a conventional PR programme.

The strengths of this study include the multicentre design, rigorous methodology, powering for an adequate sample size to test our a priori hypothesis and the intention-to-treat analysis, which limits the risk of bias. We recruited patients with severely progressed COPD using national inclusion and exclusion criteria identical to routine clinical practice for conventional outpatient hospital-based PR. Blinding was also a strength of the present study. Our assessment of outcomes was performed with documented small and acceptable measurement errors. ${ }^{21} \mathrm{~A}$ limitation of the study concerns the small but real variation in exercise content and volume among the seven hospitals delivering conventional PR, which was not possible to monitor and align. However, this is a true reflection of the real-world setting and thereby a real-world comparison. Different practical challenges to modelling, staffing and structuring PTR and differing patient acceptance of PTR in different countries, geographical regions and different types of healthcare system are limitations of the generalisability of our findings.

Proper organisation of PTR remains a challenge. It is a delicate balance in terms of decision-makers not limiting access to outpatient conventional PR and replacing it with PTR to save the costs related to buildings, equipment, transportation, etc, while endeavouring to provide an option for patients who are unable to attend the outpatient programme. From our perspective, many research questions remain unanswered regarding PTR. Future research should address subjects such as which patients are best suited to PTR and how we accommodate the increasing focus on personalised training. We need to find ways to enhance digital literacy among elderly, frail patients and discover whether the supervised or web-based tele-model is more effective as well as cost-effective. Furthermore, it is not yet known if applications will be as as good as online groups with videoconferencing. Other aspects include the role of monitoring PTR and the long-term health-related and QoL-related outcomes. Another issue regarding future studies of both PR and PTR concerns the measured outcomes of interest. As results to date regarding the traditional exercise and QoL outcomes are not convincing in patients with severe disease status, it is necessary to try a different approach. Outcomes that embrace activities of daily living and/or reduce symptoms such as dyspnoea and fatigue are warranted. In the study by Spruit $e t a l^{29}$ a composite endpoint for response to a 40-session PR programme was constructed and patients were clustered according to response profile. Interestingly, those in the 'very good responder' cluster were characterised by a worse baseline health status in comparison with the other clusters. However, it was unclear whether this multidimensional response was driven by a single outcome measure or several measures, thus calling for further research in the context of composite outcomes. 


\section{CONCLUSION}

In conclusion, supervised PTR was not superior to supervised conventional PR in increasing 6MWD. Improvements in completion of PTR compared with PR were found; however, future non-inferiority studies of the 6MWD for PTR and PR are needed to justify recommending PTR based on better adherence to the programme.

\author{
Author affiliations \\ ${ }^{1}$ Respiratory Research Unit, Department of Respiratory Medicine, Hvidovre Hospital, \\ Hvidovre, Denmark \\ ${ }^{2}$ Department of Physical and Occupational Therapy, Bispebjerg Hospital, \\ Copenhagen, Denmark \\ ${ }^{3}$ Institute for Clinical Medicine, University of Copenhagen Faculty of Health and \\ Medical Sciences, Copenhagen, Denmark \\ ${ }^{4}$ Clinical Research Center, Copenhagen University Hospital Amager and Hvidovre, \\ Hvidovre, Denmark \\ ${ }^{5}$ Department of Pulmonary Medicine, Gentofte Hospital, Hellerup, Denmark \\ ${ }^{6}$ Department of Respiratory Medicine, Bispebjerg Hospital, Copenhagen, Denmark \\ ${ }^{7}$ Department of Respiratory Medicine, Hvidovre Hospital, Hvidovre, Denmark \\ ${ }^{8}$ Department of Respiratory Medicine and Infectious Diseases, Hillerød Hospital, \\ Hillerød, Denmark \\ ${ }^{9}$ Department of Public Health, University of Copenhagen Faculty of Health Sciences, \\ Copenhagen, Denmark
}

Acknowledgements We thank the patients who participated in this study. We also thank the departments of respiratory medicine and departments of physiotherapy and occupational therapy for their commitment and for providing the necessary resources for this multicentre project to be undertaken; the steering committee for valuable discussions for protocol development and recruitment of participants and the assessors for their consistent and invaluable effort throughout the trial period.

Contributors Concept and Design of Study: All authors; Acquisition of Data: $\mathrm{HH}$, LØ, GM, ML, TK; Analysis of Data: TK, NG, HH; Drafting of Manuscript: HH; Revision of manuscript critically for important intellectual content: All authors; Approval of final manuscript: All authors.

Funding This work was supported by the Danish Lung Foundation (charitable funding), Telemedical Center Regional Capital Copenhagen (governmental funding), TrygFonden foundation (charitable funding).

Competing interests $\mathrm{HH}$ received personal grants from the Danish Lung Foundation (charitable funding), Telemedical Center Regional Capital Copenhagen (governmental funding), TrygFonden foundation (charitable funding). The grants cover expenses conducting the trial, salary and university fee for the PhD education. TB, NB, TK, TW, LO, HFA, GM, ML, AF and NG have nothing to disclose.

Patient consent for publication Not required.

Ethics approval The trial protocol was approved by the Ethics Committee of the Capital Region of Denmark (H-15019380) and the Danish Data Protection Agency ( jr.no.: 2012-58-0004).

Provenance and peer review Not commissioned; externally peer reviewed.

Data availability statement Data are available upon reasonable request. Data sharing plan: Supervised pulmonary tele-rehabilitation versus pulmonary rehabilitation in severe COPD: a randomised multicentre trial. (1) Will individual, de-identified participant data (including data dictionaries) will be shared? Yes. (2) What data in particular will be shared? Participant data that underlie the results reported in this article, after de-identification (text, tabes, figures and appendices). (3) Whether additional, related documents will be made available? Published study protocol, statistical analytics coding used in R, consent form (in Danish). (4) When and for how long the data will become/be available? Data available until 31 December 2021. (5) The criteria to access the data (including who can request access and for what types of analyses, and the name of the data repository). Proposal for data use should be addressed to Henrik.hansen.09@regionh.dk. Data access in Denmark are under very strict juristic data protection law. Any possible access or sharing demands a part application to; (1) Danish Data Protection Agency, (2) Ethics Committee of the Capital Region, (3) National Health Data Authorities. Only if the applications are approved data will be considered available for sharing. The authors will not be able to support this process and a prolonged process must be expected.

Open access This is an open access article distributed in accordance with the Creative Commons Attribution Non Commercial (CC BY-NC 4.0) license, which permits others to distribute, remix, adapt, build upon this work non-commercially, and license their derivative works on different terms, provided the original work is properly cited, appropriate credit is given, any changes made indicated, and the use is non-commercial. See: http://creativecommons.org/licenses/by-nc/4.0/.

\section{REFERENCES}

1 McCarthy B, Casey D, Devane D, et al. Pulmonary rehabilitation for chronic obstructive pulmonary disease. Cochrane Database Syst Rev 2015;2015:1-209.

2 Paneroni M, Simonelli C, Vitacca M, et al. Aerobic exercise training in very severe chronic obstructive pulmonary disease: a systematic review and meta-analysis. Am J Phys Med Rehabil 2017;96:541-8.

3 Puhan MA, Gimeno-Santos E, Cates CJ, et al. Pulmonary rehabilitation following exacerbations of chronic obstructive pulmonary disease. Cochrane Database Syst Rev 2016:12:CD005305.

4 Spruit MA, Singh SJ, Garvey C, et al. An official American thoracic Society/European respiratory Society statement: key concepts and advances in pulmonary rehabilitation. Am J Respir Crit Care Med 2013;188:e13-64.

5 Cindy Ng LW, Mackney J, Jenkins S, et al. Does exercise training change physical activity in people with COPD? A systematic review and meta-analysis. Chron Respir Dis 2012:9:17-26.

6 Keating A, Lee A, Holland AE. What prevents people with chronic obstructive pulmonary disease from attending pulmonary rehabilitation? A systematic review. Chron Respir Dis 2011:8:89-99.

7 Spruit MA, Pitta F, Garvey C, et al. Differences in content and organisational aspects of pulmonary rehabilitation programmes. Eur Respir J 2014;43:1326-37.

8 Fischer MJ, Scharloo M, Abbink JJ, et al. Drop-out and attendance in pulmonary rehabilitation: the role of clinical and psychosocial variables. Respir Med 2009;103:1564-71.

9 Milner SC, Boruff JT, Beaurepaire C, et al. Rate of, and barriers and enablers to, pulmonary rehabilitation referral in COPD: a systematic scoping review. Respir Med 2018; 137:103-14.

10 Bjoernshave B, Korsgaard J, Nielsen CV. Does pulmonary rehabilitation work in clinical practice? A review on selection and dropout in randomized controlled trials on pulmonary rehabilitation. Clin Epidemiol 2010;2:73-83.

11 Bjoernshave B, Korsgaard J, Jensen C, et al. Participation in pulmonary rehabilitation in routine clinical practice. Clin Respir J 2011;5:235-44.

12 Rochester CL, Vogiatzis I, Holland AE, et al. An official American thoracic Society/ European respiratory Society policy statement: enhancing implementation, use, and delivery of pulmonary rehabilitation. Am J Respir Crit Care Med 2015;192:1373-86.

13 Bourne S, DeVos R, North M, et al. Online versus face-to-face pulmonary rehabilitation for patients with chronic obstructive pulmonary disease: randomised controlled trial. BMJ Open 2017;7:e014580.

14 Chaplin E, Hewitt S, Apps L, et al. Interactive web-based pulmonary rehabilitation programme: a randomised controlled feasibility trial. BMJ Open 2017;7:e013682

15 Bernocchi P, Vitacca M, La Rovere MT, et al. Home-based telerehabilitation in older patients with chronic obstructive pulmonary disease and heart failure: a randomised controlled trial. Age Ageing 2018;47:82-8.

16 Tsai LLY, MCNamara RJ, Moddel C, et al. Home-based telerehabilitation via real-time videoconferencing improves endurance exercise capacity in patients with COPD: the randomized controlled TeleR study. Respirology 2017:22:699-707.

17 Hansen $\mathrm{H}$, Bieler T, Beyer N, et al. COPD online-rehabilitation versus conventional COPD rehabilitation - rationale and design for a multicenter randomized controlled trial study protocol (CORe trial). BMC Pulm Med 2017;17:140.

18 Capital Region D. Forløbsprogram RegionH (Revideret Okt. 2015).Pdf 2015.

19 Danish National board of Health. National Klinisk Retningslinje for Rehabilitering Af Patienter Med KOL. Copenhagen, Denmark, 2018. https://www.sst.dk/da/udgivelser/ 2018/ /media/AD2FF426014943D983E0D7B937B356B9.ashx

20 Mølsted S, Dall CH, Hansen H, et al. Anbefalinger Til Superviseret Fysisk Traening Af Mennesker Med Type 2-Diabetes, KOL Og Hjerte-Kar-Sygdom. Hillerød, 2012. https:// www.regionh.dk/til-fagfolk/Sundhed/Tvaersektorielt-samarbejde/kronisk-sygdom/ Publishinglmages/Sider/Rehabilitering/2206717238_RapportRHLauraJenniferMunkø_ low.pdf

21 Hansen $\mathrm{H}$, Beyer $\mathrm{N}$, Frølich $\mathrm{A}$, et al. Intra- and inter-rater reproducibility of the 6-minute walk test and the 30-second sit-to-stand test in patients with severe and very severe COPD. Int J Chron Obstruct Pulmon Dis 2018:13:3447-57.

22 Danish Society of Respiratory Medicine. Lungefunktionsstandard Spirometri Og Peakflow, 2007. Available: https://www.lungemedicin.dk/fagligt/klaringsrapporter/ 5-Ifu-standard/file.html

23 Holland AE, Hill CJ, Rasekaba T, et al. Updating the minimal important difference for six-minute walk distance in patients with chronic obstructive pulmonary disease. Arch Phys Med Rehabil 2010;91:221-5.

24 Puhan MA, Chandra D, Mosenifar Z, et al. The minimal important difference of exercise tests in severe COPD. Eur Respir J 2011:37:784-90.

25 Holland AE, Spruit MA, Troosters T, et al. An official European respiratory society/ American thoracic society technical standard: field walking tests in chronic respiratory disease. Eur Respir J 2014:44:1428-46.

26 Molenberghs G, Kenward MG. Missing data in clinical studies. Wiley Blackwell, 2007. 
27 Holland AE, Mahal A, Hill CJ, et al. Home-based rehabilitation for COPD using minimal resources: a randomised, controlled equivalence trial. Thorax 2017;72:57-65.

28 Horton EJ, Mitchell KE, Johnson-Warrington V, et al. Comparison of a structured home-based rehabilitation programme with conventional supervised pulmonary rehabilitation: a randomised non-inferiority trial. Thorax 2018;73:29-36.

29 Spruit MA, Augustin IM, Vanfleteren LE, et al. Differential response to pulmonary rehabilitation in COPD: multidimensional profiling on behalf of the CIRO+ rehabilitation network. Eur Respir J 2015;46:1625-35.
30 Dechartres A, Boutron I, Trinquart L, et al. Single-center trials show larger treatment effects than multicenter trials: evidence from a meta-epidemiologic study. Ann Intern Med 2011;155:39-52.

31 Boutou AK, Tanner RJ, Lord VM, et al. An evaluation of factors associated with completion and benefit from pulmonary rehabilitation in COPD. BMJ Open Respir Res 2016;1.

32 Danish Regions. Danish Registry for Chronic Obstructive Pulmonary Disease - National Annual Report 2017. Aarhus, Denmark, 2017. www.rkkp.dk

33 Tudor-Locke C, Craig CL, Brown WJ, et al. How many steps/day are enough? for adults. Int I Behav Nutr Phys Act 2011;8:79. 